\title{
Photorheological fluids of azobenzene polymers for lubrication regulation
}

\author{
Shuangxi TANG ${ }^{1}$, Shayu $\mathbf{L I}^{2}$, Liran MA ${ }^{1, *}$, Yu TIAN ${ }^{1, *}$ \\ ${ }^{1}$ State Key Laboratory of Tribology, Department of Mechanical Engineering, Tsinghua University, Beijing 100084, China \\ ${ }^{2}$ College of Chemistry and Chemical Engineering, Chongqing University, Chongqing 401331, China \\ Received: 12 March 2021 / Revised: 18 May 2021 / Accepted: 21 May 2021 \\ (C) The author(s) 2021.
}

\begin{abstract}
Fluid viscosity is ubiquitous property and is of practical importance in intelligent fluids, industrial lubrication, and pipeline fluid transportation. Recently, there has been a surging interest in viscosity regulation. Here, we have developed a group of photorheological fluids by utilizing azobenzene polymers with a light-induced microstructure transformation. In this work, a photosensitive polymer with 4,4'-bis-hydroxyazobenzene as the main chain was designed and synthesized as a pivotal functional material. The sufficiently large structural difference under ultraviolet and near-infrared light makes it possible to regulate the viscosity of a polyethylene glycol solution. The viscosity of the photosensitive rheological fluids under ultraviolet light radiation is found to be up to $45.1 \%$ higher than that under near-infrared light radiation. To explore this intelligent lubricating technology, the friction regulation of ceramic sliding bearings was investigated utilizing photosensitive rheological fluids. Reversible friction regulation with a ratio of up to 3.77 has been achieved by the alternative irradiation of near-infrared and ultraviolet light, which can be attributed to the differences in mechanical properties and molecular structures under ultraviolet and near-infrared light according to both simulations and experiments. Such photorheological fluids will have promising applications in controllable lubrication, intelligent rheological fluids, and photosensitive dampers.
\end{abstract}

Keywords: photorheological fluids; viscosity; azobenzene polymer; lubrication

\section{Introduction}

The viscosity of fluids is closely tied to industrial production and daily life, such as in navigation [1, 2], pipe flows [3], mechanical lubricants [4-11], water conservancy, and biorheology [12, 13]. In particular, with the development of intelligent fluids such as electrorheological fluids [14, 15], magnetorheological fluids [16-20], $\mathrm{pH}$-sensitive fluids [21], photorheological fluids [22-24], and other stimuli-responsive fluids $[25,26]$, these intelligent fluids are attracting extensive attention as smart fluid dampers for vehicle [27, 28], oil and gas pipeline transportation [29], in wafer surface polishing [30, 31], and friction regulation [5, 32-36]. However, some intelligent fluids have disadvantages, such as in electrochemical corrosion in electrorheological fluids. In addition, irreversible transformation properties, high cost and environmental pollution have blocked the development of other stimuli-responsive fluids [21,37]. As the application of intelligent fluids has increased, new kinds of liquids are urgently needed. Among all kinds of stimuli, light stimuli have a series of advantages, including convenience, cleanliness, biocompatibility, and fast response [38, 39]. The light field shows wide potential applications in intelligent materials and mechanical systems, such as for hydrophilic/hydrophobic wetting surfaces [40, 41], light-sensitive hydrogels [42], and light-induced mechanical arms [43,44]. Therefore, light is considered an ideal outside stimulus to regulate the viscosity of photosensitive intelligent fluids.

\footnotetext{
* Corresponding authors: Liran MA, E-mail: maliran@tsinghua.edu.cn; Yu TIAN, E-mail: tianyu@mail.tsinghua.edu.cn
} 
Recently, a class of organic photosensitive materials has aroused increasing interest on account of their significant structural differences and responsive performance. To date, great efforts have been dedicated to intelligent materials [45-47], including diarylethene, fulgide, azobenzene, spiropyran, carbon nanotubes, and graphene oxide. These photosensitive materials are widely used in flexible robots [48], liquid transportation [40], and photoforce sensors [49]. Among these external stimuli-responsive materials, azobenzene and its derivatives have attracted the most attention due to large changes in both their geometry and molecular length under ultraviolet (UV)/visible/ thermal stimuli [50-53]. Azobenzene has two isomers, trans isomers (under visible/thermal/near-infrared (NIR) light) and cis isomers (under UV light). The molecular length of the cis isomer is $38.8 \%$ shorter than that of the trans isomer. Most atoms of the trans isomer are coplanar, while those of the cis isomer are noncoplanar [53, 54]. In addition, the photoisomerization of azobenzene is reversible, which makes it possible to prepare reusable photosensitive rheological fluids. Although a single azobenzene molecule has obvious structural differences between the trans isomer and cis isomer, the differences at the angstrom scale are not effective enough to influence the macroscale performance of photosensitive rheological fluids. Therefore, magnifying the differences between the cis and trans isomer is a simple and efficient way to solve the problem. A photosensitive polymer with azobenzene as the main chain is promising to satisfy these requirements. Organic photosensitive azobenzene polymers as optical switching materials provide the advantages of being green, efficient, saving and intelligent but present challenges in developing excellent photosensitive rheological fluids.

In this work, we designed a kind of photosensitive polymer by integrating 4,4'-bis-hydroxyazobenzene as a photosensitizer into a polycondensation polymer. Interestingly, the photosensitive azobenzene polymer exhibits two totally different morphologies: One conformation is a linear chain under NIR light, and the other conformation is a serrated chain under UV light. In addition, the mechanical properties, including the Young's modulus and shear modulus, are also different under UV and NIR light. The viscosity of the photosensitive rheological fluid under UV light is up to $45.1 \%$ higher than that under NIR light. Photosensitive rheological fluids have shown obvious reversible regulation of the friction of ceramic sliding bearings. The maximum friction coefficients could be changed by approximately 3.8-fold. These results have potential applications in intelligent fluid braking, microfluid transportation, and a wide range of fields requiring external stimuli-responsive viscosity.

\section{Materials and methods}

\subsection{Materials}

Chemicals including azobenzene derivative and hexamethylenene diisocyanate were purchased from Tokyo Chemical Industry and Sinopharm Chemical Reagent Corporation. Other reactants and catalysts, such as polyglycol 200/300/400, tetrahydrofuran (THF), and $\mathrm{Bu}_{2} \mathrm{Sn}$ dilaurate, were obtained from Aldrich. The purity of all of these reagents is AR grade.

In this work, polymer materials were synthesized to achieve photoresponsive properties via azobenzene functional groups. 4,4'-bis-hydroxyazobenzene was particularly important to realize the folding and stretching of the polymer chains corresponding to the cis isomer and trans isomer conformations. The polymer was the polycondensate of 4,4'-bishydroxyazobenzene and hexamethylenene diisocyanate. Hexamethylene diisocyanate played a connecting role in the polymer chain. The synthesis process is described in the Electronic Supplementary Material (ESM). The final product was a brown amorphous powder with chemical name poly (4,4'-bis-hydroxyazobenzene hexamethylene diisocyanate) (P-AZO-HDI).

\subsection{Characterizations of P-AZO-HDI}

The mass spectra of the P-AZO-HDI polymer were measured by gas chromatography mass spectrometry (GC/MS-QP2010). The P-AZO-HDI polymer was dissolved in acetone to obtain the tested samples. The IR absorption spectra were characterized by a Fourier transform infrared spectrometer (Bruker, Horiba, Germany). During the Infrared spectra measurements, the samples were prepared by the squash method to form a submillimeter thick film, and the test was 
performed under atmospheric conditions at room temperature. The UV-visible absorption spectra were recorded on a UV-visible spectrophotometer (UV-2700, Shimadzu, Japan). The samples were composed of $1 \times 10^{-5} \mathrm{~mol} \cdot \mathrm{L}^{-1} \mathrm{P}$-AZO-HDI and N-methyl pyrrolidone (NMP) solution. The morphology of the powder or film formed of P-AZO-HDI was observed by scanning electron microscopy (SEM).

\subsection{Photoisomerization experiments}

To characterize the light-induced transition between the cis and trans isomers, P-AZO-HDI was illuminated by a mercury lamp (Philips, $\lambda=210-380 \mathrm{~nm}$ ). NIR irradiation was carried out by using an IR-250 RH lamp (Philips, $\lambda=760-5,000 \mathrm{~nm}$ ). Azobenzene can transform from the cis isomer into the trans isomer under the infrared thermal effect. Here, NIR light was used to achieve a rapid heating goal.

\subsection{Viscosity measurements}

The light-controlled viscosities of the photosensitive fluids were measured on an Anton Paar Physica (MCR302) rheometer. The test temperature was at an ambient temperature of $25^{\circ} \mathrm{C}$. The cone-plate measuring system was selected to measure the viscosity. The diameter of the cone was $50 \mathrm{~mm}$, and the coneplate gap was equal to $103 \mu \mathrm{m}$. The angle of the cone was $179^{\circ}$. The shear rates ranged from 1 to $300 \mathrm{~s}^{-1}$, and the total test time was approximately $3 \mathrm{~min}$ for each sample. To explore the photoresponsive viscosity, each sample was adequately irradiated by UV/NIR light before testing. During the viscosity test, the samples and rheometer were sheltered to avoid the influence of ambient light.

\subsection{Light-controlled friction measurements}

The friction measurements of ceramic bearings with pure sliding system were conducted on a UMT3 tribometer. $\mathrm{A} \mathrm{Si}_{3} \mathrm{~N}_{4}$ ceramic bearing material was selected to achieve the light-controlled friction regulation. A ball-on-disk configuration was adopted to simulate the working conditions of the bearing. The sphere was a $\mathrm{Si}_{3} \mathrm{~N}_{4}$ ball with a diameter of $10.7 \mathrm{~mm}$, and the flat surface was a thin slice of $\mathrm{SiO}_{2}$. The test speeds ranged from 0 to $330 \mathrm{~mm} \cdot \mathrm{s}^{-1}$, and the speed interval was $5.23 \mathrm{~mm} \cdot \mathrm{s}^{-1}$. The total test time was approximately $10 \mathrm{~min}$, and the measurements were performed at room temperature $\left(25^{\circ} \mathrm{C}\right)$. The load was set to $1 \mathrm{~N}$, and a $500 \mathrm{~g}$ sensor was selected. As calculated by Hertzian elastic contact theory, the maximum Hertzian contact pressure was 289.1 MPa and the Hertzian contact radius was $40.6 \mu \mathrm{m}$. The photosensitive fluid samples were the same as the viscosity-tested solutions, which included pure PEG200, PEG300, PEG400, and their corresponding solutions containing $2 \mathrm{wt} \%$ P-AZO-HDI. These samples were illuminated by NIR light or UV light for $2 \mathrm{~min}$. After irradiation, the solutions were stored in a dark environment until the temperature recovered to $25^{\circ} \mathrm{C}$.

\subsection{Elastic modulus measurements}

The Young's modulus of P-AZO-HDI flakes under UV/NIR light illumination was obtained by Atomic Force Microscope (AFM) in situ measurements under ambient conditions with shading treatment. The flakes of the P-AZO-HDI polymer were prepared by compression molding. First, $6 \mathrm{mg}$ P-AZO-HDI powder was weighed and put into a square mold. Then, a $0.3 \mathrm{MPa}$ pressure was maintained for $10 \mathrm{~min}$ on the mold. Finally, submillimeter transparent flakes were obtained by careful demolding. The flakes were fixed on a glass substrate, and the force curves were measured in situ under different irradiation conditions. In this work, a conical silicon probe was selected to survey the elastic modulus. The Young's modulus was obtained by fitting the force curves with the Hertz/Sneddon contact model. The fitting process can be found in the ESM.

\subsection{Simulation calculation}

The P-AZO-HDI property calculations were simulated by Material Studio 2017. The photoresponsive P-AZO-HDI polymer had two kinds of isomers, the trans isomer (NIR), and cis isomer (UV). There were three tasks, including chain morphology, shear modulus, and Young's modulus, for each isomer. The method to simulate the morphology was to optimize the geometric structure of the cis and trans isomers. Here, the end-to-end distance and radius of gyration of the P-AZO-HDI polymer were selected to characterize the morphology differences between the cis and trans isomers. Taking the degree of polymerization into 
account, a series of polymers were simulated to observe the morphology of the cis/trans polymer. The shear modulus and Young's modulus were calculated by constructing an amorphous cell. The cell was formed with a certain proportion of polymers with different polymerization degrees. In this simulated calculation, the crystal building tool and Forcite tool were applied to optimize the geometry and calculate the mechanical properties. The detailed modeling and simulation process are listed in the ESM.

\section{Results and discussions}

\subsection{Photoresponsive polymer P-AZO-HDI}

The transformation between the cis isomer and trans isomer of azobenzene plays a decisive role in the lightcontrolled viscosity. The trans isomer can be converted to a cis isomer by irradiation with UV light, and recovers to the initial state when triggered by visible/thermal (NIR) stimuli, as shown in Fig. 1(a). The cis isomer and trans isomer of azobenzene have obvious differences in molecular length and structural conformation. To further magnify the differences in azobenzene properties, azobenzene was introduced into the main chain of the P-AZO-HDI polymer. The powder morphology of P-AZO-HDI was flowers in clusters, as shown in Fig. 1(b). The UV-visible absorption spectrum of P-AZO-HDI was measured to determine the lightresponsive wavelength, which was important for the design and application of light-controlled viscosity. There were two absorption peaks in the UV-visible spectrum of azobenzene. A strong peak was at $365 \mathrm{~nm}$, and a weak peak was at $440 \mathrm{~nm}$. In the UV-visible spectrum of the polymer, the $440 \mathrm{~nm}$ absorption peak was too weak to be submerged. The UV-visible spectrum of P-AZO-HDI with NMP as the solvent had a strong absorption peak at $365 \mathrm{~nm}$ in the primary state, as shown in Fig. 1(c). Then, after exposure to UV light for $2 \mathrm{~min}$, the $365 \mathrm{~nm}$ absorption peak was quickly reduced. It was basically recovered to the initial value via illumination with NIR light for 2-5 min. In addition, the cis isomer can be converted to a trans isomer at temperatures higher than $80^{\circ} \mathrm{C}$. NIR light had an obvious advantage in its rapid heating to trigger the cis-trans transformation due to its excellent thermal effect. The results demonstrated that P-AZO-HDI had good photoresponsive properties triggered by NIR/UV light.

To analyze the functional groups of the P-AZO-HDI polymer, IR absorption spectroscopy was conducted, as shown in Fig. 1(d). The stretching vibration peaks of the $\mathrm{N}-\mathrm{H}$ bonds (new bonds) and $\mathrm{C}-\mathrm{H}_{2}$ bonds were 3,331 and $2,933 \mathrm{~cm}^{-1}$, respectively. The wavenumbers (a)

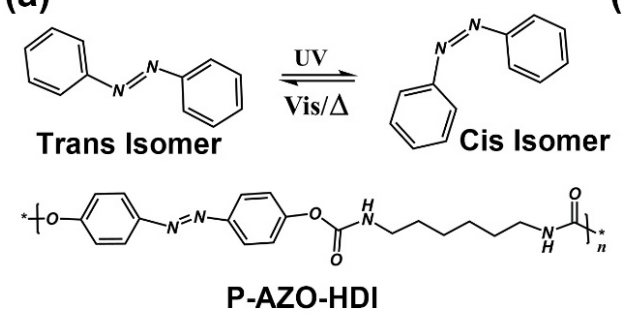

(b)

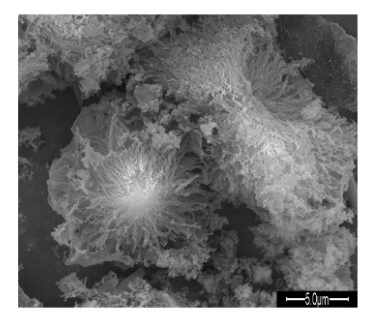

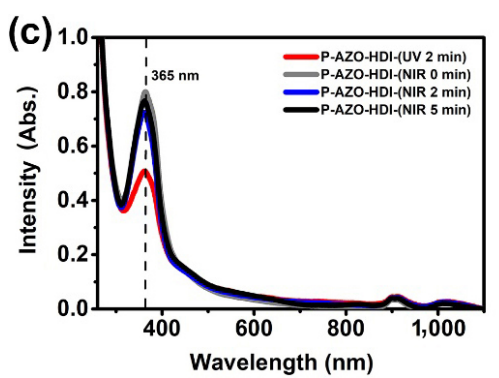

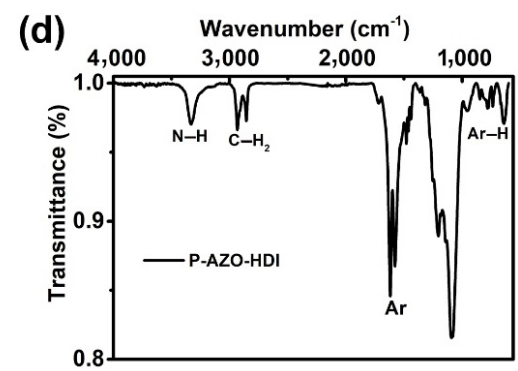

Fig. 1 Basic characterization of the photosensitive polymer P-AZO-HDI. (a) Chemical structure of P-AZO-HDI and photoresponsive mechanism of azobenzene; (b) SEM image of the P-AZO-HDI powder; (c) UV-visible absorption spectra of the P-AZO-HDI polymer (The UV absorbed peak was at $365 \mathrm{~nm}$ ); and (d) IR absorption spectrum of P-AZO-HDI (IR: $3,331 \mathrm{~cm}^{-1}\left(\mathrm{~m} ; v_{\mathrm{s}}(\mathrm{NH})\right), 2,933 \mathrm{~cm}^{-1} \& 2,856 \mathrm{~cm}^{-1}$ $\left.\left(\mathrm{m} ; \mathrm{v}_{\mathrm{s}}\left(\mathrm{CH}_{2}\right)\right), 1,617 \mathrm{~cm}^{-1} \& 1,577 \mathrm{~cm}^{-1}\left(\mathrm{~s} ; \mathrm{v}_{\mathrm{c}=\mathrm{c}}(\mathrm{Ar})\right), 645-950 \mathrm{~cm}^{-1}(\mathrm{~m} ; \omega(\mathrm{Ar}-\mathrm{H}))\right)$. 
at 1,617 and $1,577 \mathrm{~cm}^{-1}$ were the vibration peaks of the benzene skeleton. The wavenumbers from 645 to $950 \mathrm{~cm}^{-1}$ were the stretching vibration peaks of the $\mathrm{Ar}-\mathrm{H}$ bonds. The full IR spectral interpretation can be found in Fig. S1(a) in the ESM. P-AZO-HDI was the polycondensate of an azobenzene derivative and hexamethylene diisocyanate, as shown in Fig. 1(a) and Scheme $\mathrm{S} 1$ in the ESM. The $\mathrm{C}-\mathrm{H}_{2}$ bonds were from hexamethylene diisocyanate. The benzene skeleton and $\mathrm{Ar}-\mathrm{H}$ bonds were from the azobenzene derivative. There were characteristic peaks of the two reactants in the IR spectrum of P-AZO-HDI. The results indicated that the polycondensation reaction was successfully ongoing. In addition, the mass spectrum (Fig. S1(b) in the ESM) was measured to determine the degree of polymerization of P-AZO-HDI. The degree of polymerization was spread across 7 to 20 , and most polymers fell within 7 to 12 . By roughly simulating the polymer structures, the molecular lengths of the P-AZO-HDI polymer were distributed from 10 to $40 \mathrm{~nm}$. Therefore, the P-AZO-HDI was successfully synthesized through experimental and analytical results.

\subsection{Light-controlled viscosity}

The light-controlled viscosities of the P-AZO-HDI and PEG200/300/400 solutions were measured on a cone-on-plate system. The tested shear rates ranged from 0 to $300 \mathrm{~s}^{-1}$ at $25{ }^{\circ} \mathrm{C}$. The viscosities of four photorheological fluids (PEG300 and P-AZO-HDI) of different concentrations were tested to determine the best concentration. The variation rate $(\Delta)$ of the viscosity was defined as the increase in viscosity divided by the viscosity under NIR light. The variation rate of the viscosity increased as the P-AZO-HDI content increased, as shown in Fig. 2(a). The average variation rates were $5.1 \%$ for $0.5 \mathrm{wt} \%$ fluid, $7.5 \%$ for $1 \mathrm{wt} \%$ fluid, $10.7 \%$ for $1.5 \mathrm{wt} \%$ fluid, and $25.4 \%$ for $2 \mathrm{wt} \%$ fluid. The solubility of P-AZO-HDI in polyethylene glycol is close to $2 \mathrm{wt} \%$ at room temperature. The viscosities of $2 \mathrm{wt} \%$ P-AZO-HDI and PEG300 changed from 126.92 to $87.95 \mathrm{mPa} \cdot \mathrm{s}$ under UV light as the shear rates increased from 0 to $300 \mathrm{~s}^{-1}$. The viscosities of $2 \mathrm{wt} \%$ P-AZO-HDI and PEG300 ranged from 88.28 to $75.28 \mathrm{mPa} \cdot \mathrm{s}$ when exposed to NIR light, as shown in Fig. 2(b). At most, there was a $43.8 \%$ rate of change. The dripping test of PEG300 and $2 \mathrm{wt} \%$ P-AZO-HDI solution under UV and NIR irradiation was recorded by camera. As shown in Fig. 2(c), after irradiation with UV or NIR light, $1 \mathrm{~mL}$ of the fluid was added to the pipettes. The dripping duration time of the NIRirradiated liquid was $13.0 \mathrm{~s}$, while the dripping (a)

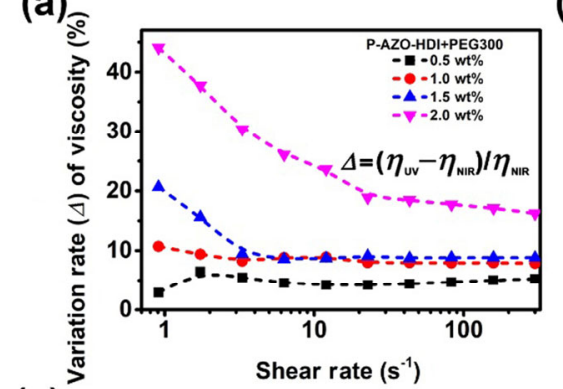

(b)

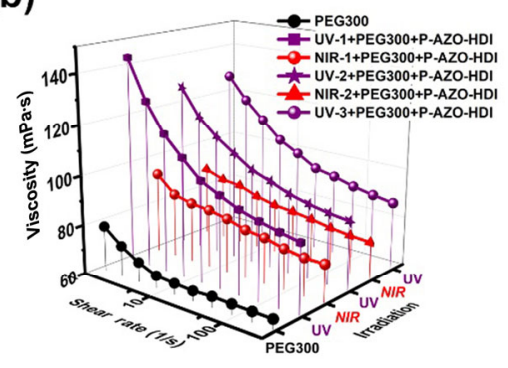

(c)

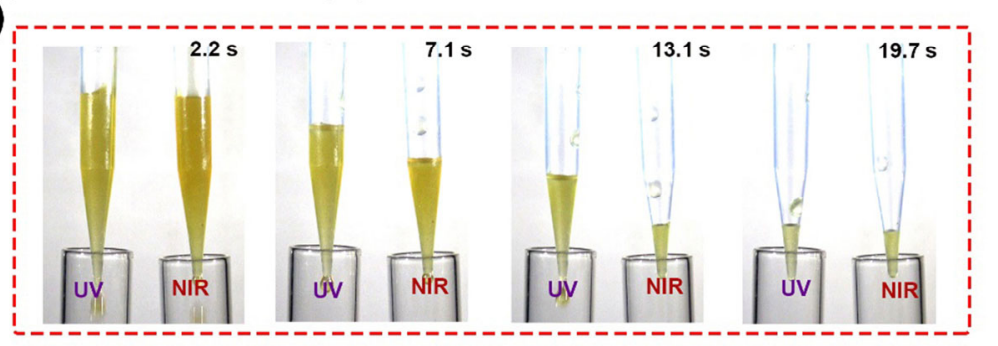

Fig. 2 Light-controlled viscosities of P-AZO-HDI and PEG300 solutions triggered by NIR/UV light. (a) $\Delta$ of photoresponsive PEG200 solutions with P-AZO-HDI concentrations of $0.5,1.0,1.5$, and $2.0 \mathrm{wt} \%$; (b) reversible light-controlled viscosity transformations of $2 \mathrm{wt} \%$ P-AZO-HDI and PEG300 solutions; and (c) photosensitive rheological fluid dripping test of $1 \mathrm{~mL} 2 \mathrm{wt} \% \mathrm{P}-\mathrm{AZO}-\mathrm{HDI}$ and PEG300 solutions. 
duration time of the UV-irradiated liquid was $19.5 \mathrm{~s}$. This meant that the viscous forces of the fluids under UV light were higher than those under NIR light. Repeating the reversible light-controlled viscosity experiments several times, the viscosities of UV irradiation were typically higher than those of NIR light.

Most solutions of $2 \mathrm{wt} \%$ P-AZO-HDI mixed with polyethylene glycol (Figs. 2(b), 3(a), and 3(b)) behaved as non-Newtonian fluids with shear thinning properties. However, pure polyethylene glycol essentially maintained Newtonian fluid properties. The viscosities of solutions irradiated by UV light were typically higher than those irradiated by NIR light. At low shear rates, the viscosity difference was greater. When the shear rates were higher than $30 \mathrm{~s}^{-1}$, the viscosity differences remained relatively stable. The viscosities of pure PEG200, pure PEG300, and pure PEG400 were $45.78 \pm 2.057,66.97 \pm 4.816$, and $93.35 \pm 0.385 \mathrm{mPa} \cdot \mathrm{s}$, respectively. The viscosities of $2 \mathrm{wt} \% \mathrm{P}-\mathrm{AZO}-\mathrm{HDI}$ and PEG200 exposed to UV light were 20-65 mPa.s higher than those irradiated by NIR light, as shown in Fig. 3(a). The change extent of the viscosities was up to $45.1 \%$. The viscosities of the $2 \mathrm{wt} \%$ P-AZO-HDI and PEG400 solution irradiated by UV light were 10-17 $\mathrm{mPa} \cdot \mathrm{s}$ higher than those under NIR light, as shown in Fig. 3(b). The regulated rates ranged from $11.1 \%$ to $16.4 \%$. In addition, the viscosities of the P-AZO-HDI and polyethylene glycol solutions were reversibly transformed by alternating UV and NIR light irradiation, as shown in Figs. 2(b) and S2(a), and S2(b) in the ESM. The results of the fatigue tests indicated that the light-controlled viscosities of P-AZOHDI and polyethylene glycol had a stable and obvious regulation in the photosensitive rheological fluids.
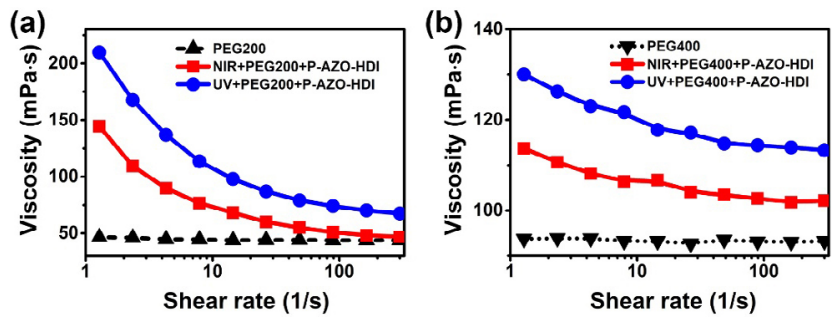

Fig. 3 Light-controlled viscosities of $2 \mathrm{wt} \%$ P-AZO-HDI dissolved in (a) PEG200 solution and (b) PEG400 solution.

\subsection{Mechanical properties of P-AZO-HDI}

The mechanical and structural properties of the
P-AZO-HDI polymer played a significant role in the light-controlled viscosity regulation. The Young's modulus of P-AZO-HDI was determined by AFM in situ measurements. A diagram of the light-controlled Young's modulus measurement is shown in Fig. 4(a). The test sample was a thin film made by the squash method. There were two force curves obtained by extending and retracting the motions of the AFM probe. Here, the extending force curve was selected to fit as described in the ESM. A 160AC-NA standard silicon probe was adopted to measure force curves, as shown in Fig. S3(a) in the ESM. The fitting modulus was the Hertz/Sneddon elastic contact modulus, as shown in Fig. S3(b) in the ESM. The Young's modulus was 5.304 GPa when exposed to NIR light and 2.634 GPa when irradiated by UV light as shown in Fig. 4(b). The Young's modulus was repeated several times, and the average Young's modulus was 7.975 \pm 2.357 GPa under NIR light and 3.039 \pm 0.351 GPa under UV light, as shown in Fig. 4(c) and Table S2 in the ESM. The simulation results supported the Young's modulus measured by AFM. The Young's moduli of the simulation results were $0.526 \mathrm{GPa}$ for the trans isomer (NIR) and 0.153 GPa for the cis isomer (UV), as shown in Fig. 4(d). There was a regular pattern in which the Young's modulus of the trans isomer (NIR) was significantly higher than that of the cis isomer (UV).

According to the simulated results of the polymer structures, there were obvious differences between the molecular morphologies of the trans isomer (NIR) and cis isomer (UV). The trans isomer polymers were mainly long linear-chain structures, while the cis isomer polymers were serrated-chain structures, as shown in Fig. S3(f) in the ESM. Here, the terminal distance and radius of gyration of the P-AZO-HDI polymers were selected to investigate the difference between the trans and cis polymers. The terminal distance of the polymer was defined by the length between the first atom and the last atom of the molecule. Molecules with longer terminal distance had better straightness at the same degree of polymerization. The terminal distances of the trans isomer (NIR) were generally 4.17-11.62 $\mathrm{nm}$ longer than that of the cis isomer (UV) as the degree of polymerization increased from 7 to 20, as shown in Fig. 4(e) and Table S1 in the ESM. There was a different of up to $33 \%$ in molecular length 

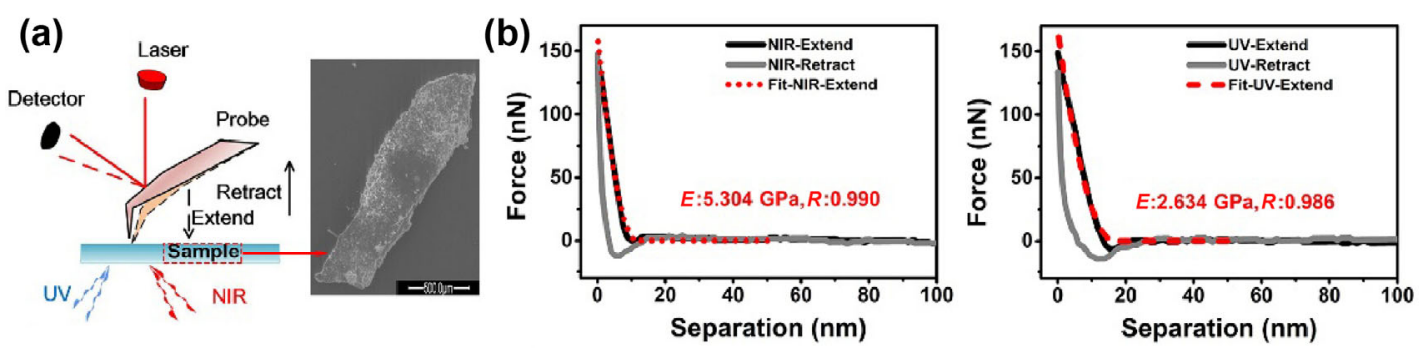

(c)

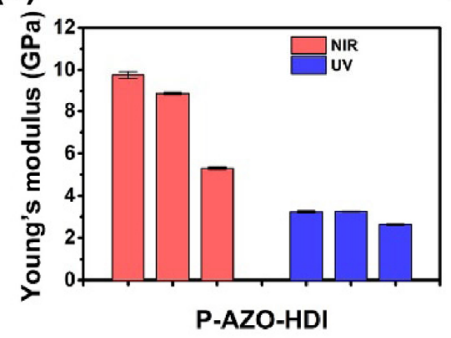

(d)

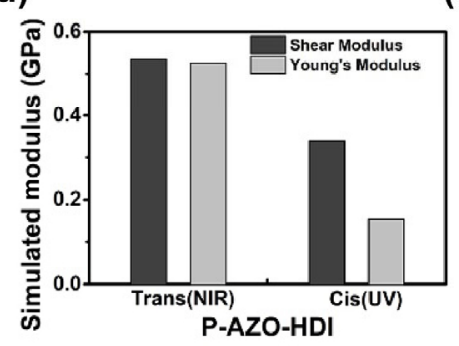

(e)

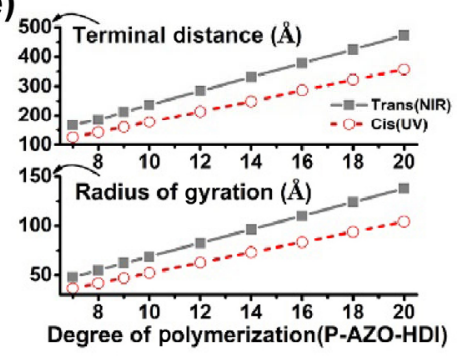

Fig. 4 Mechanical performance and molecular simulation results of P-AZO-HDI. (a) Diagram of the light-controlled Young's modulus measurement by AFM; (b) fitting force curves of P-AZO-HDI (The Young's modulus was 5.304 GPa under NIR light and 2.634 GPa under UV light); (c) statistical chart of the reversible Young's modulus of the P-AZO-HDI film (The average Young's modulus was $7.975 \pm 2.357 \mathrm{GPa}$ under NIR light and $3.039 \pm 0.351 \mathrm{GPa}$ under UV light); (d) simulated results of mechanical properties (The Young's moduli were $0.526 \mathrm{GPa}$ for the trans isomer (NIR) and $0.153 \mathrm{GPa}$ for the cis isomer (UV). The shear moduli were $0.534 \mathrm{GPa}$ for the trans isomer and $0.339 \mathrm{GPa}$ for the cis isomer); and (e) results of the molecular conformation simulation (Light-controlled molecular structures with a series of degrees of polymerization were characterized by terminal distance and radius of gyration).

between the trans and cis polymers at the same degree of polymerization. In general, a smaller radius of gyration represented a more twisted polymer. The radius of gyration of the trans isomer (NIR) was up to $24 \%$ larger than that of the cis isomer (UV). The simulated results indicated that the trans isomer was straighter than the cis isomer. In addition, the simulated shear modulus of the trans isomer polymer was $0.534 \mathrm{GPa}$ while that of the cis isomer polymer was $0.339 \mathrm{GPa}$, as shown in Fig. 4(e). The mechanical properties and molecular structure differences between the trans and cis isomers provided the possibility of achieving light-controlled viscosity.

\subsection{Friction regulation by photosensitive rheological fluids}

According to the Hamrock-Dowson film thickness formula (Eqs. (S5) and (S6) in the ESM), the film thickness of the contacted areas will increase $0.67 \%$ as the viscosity increases $1 \%$. Therefore, the lightcontrolled viscosity of rheological fluids had an important influence on the friction of contact area, especially in the boundary lubrication and mixed lubrication. The photosensitive rheological fluid is potential to application in the light-induced intelligent braking of some devices, such as dampers, gears, and bearings. Here, the friction measurement of ceramic sliding bearings (Fig. 5(a)) was conducted on a universal mechanical tester type 3 (UMT3) adopting a point contact mode. The silicon nitride sliding bearing and the diagram of light-controlled friction are shown in Fig. 5(b). The COF of pure PEG200/300/400 was also measured, as shown in Fig. 5(c). The COF of pure PEG200/300/400 first decreased and then remained relatively stable as the test speeds increased from 0 to $330 \mathrm{~mm} \cdot \mathrm{s}^{-1}$. The coefficients of fiction (COFs) that changed with test speeds from 0 to $330 \mathrm{~mm} \cdot \mathrm{s}^{-1}$ were recorded under NIR/UV light. The COF of the $2 \mathrm{wt} \%$ P-AZO-HDI and polyethylene glycol solutions had obvious differences after irradiation by NIR/UV light, especially when the test speeds were lower than $180 \mathrm{~mm} \cdot \mathrm{s}^{-1}$, as shown in Fig. 5(d). However, the lightcontrolled friction lost effectiveness at speeds greater than $180 \mathrm{~mm} \cdot \mathrm{s}^{-1}$. It was conjectured that the lubricated state had been transformed from mixed lubrication to elastohydrodynamic lubrication at a speed of $180 \mathrm{~mm} \cdot \mathrm{s}^{-1}$. Because the thickness of lubricated film was about $15 \mathrm{~nm}$ at the speed of $150 \mathrm{~mm} \cdot \mathrm{s}^{-1}$ and 
the comprehensive surface roughness was $5 \mathrm{~nm}$. In elastohydrodynamic lubrication, the film thickness separates the contacted interface by a thick fluid layer. Therefore, the COFs under UV and NIR light were almost the same at speeds greater than $180 \mathrm{~mm} \cdot \mathrm{s}^{-1}$. The COFs under NIR light were much higher than those under UV light at speeds below $180 \mathrm{~mm} \cdot \mathrm{s}^{-1}$, as shown in Figs. 5(e)-5(g). Whether under UV or NIR light irradiation, the COF change trend of P-AZO-HDI in different polyethylene glycols followed similar rules, decreasing first and then increasing slowly as the speed increased.

Here, the COF ratio was defined by the COF under NIR light divided by that under UV light at the same speed. The COF ratio was selected to measure the regulating performances of the photosensitive rheological fluids. The maximum $\mathrm{COF}$ ratios were 3.77 in PEG200 and $2 \mathrm{wt} \%$ P-AZO-HDI solution at the speed of $68 \mathrm{~mm} \cdot \mathrm{s}^{-1}, 2.35$ in PEG300 and $2 \mathrm{wt} \%$ $\mathrm{P}-\mathrm{AZO}-\mathrm{HDI}$ solution at the speed of $26 \mathrm{~mm} \cdot \mathrm{s}^{-1}$, and 2.85 in PEG400 and 2 wt\% P-AZO-HDI solution at the speed of $10 \mathrm{~mm} \cdot \mathrm{s}^{-1}$, as shown in Fig. 5(d). The COF change ranges were $20 \%-100 \%$ in other works of light-induced friction $[5,55,56]$. Further studies of light-controlled friction fatigue tests showed that the friction regulations underwent reversible transformation under UV and NIR light, as shown in Fig. 5(h). The COF of PEG200 and $2 \mathrm{wt} \%$ P-AZO-HDI tended to alternate between $0.056 \pm 0.0098$ (NIR) and $0.015 \pm 0.0066$ (UV) at the speed of $68 \mathrm{~mm} \cdot \mathrm{s}^{-1}$. The COF of PEG300 (a)
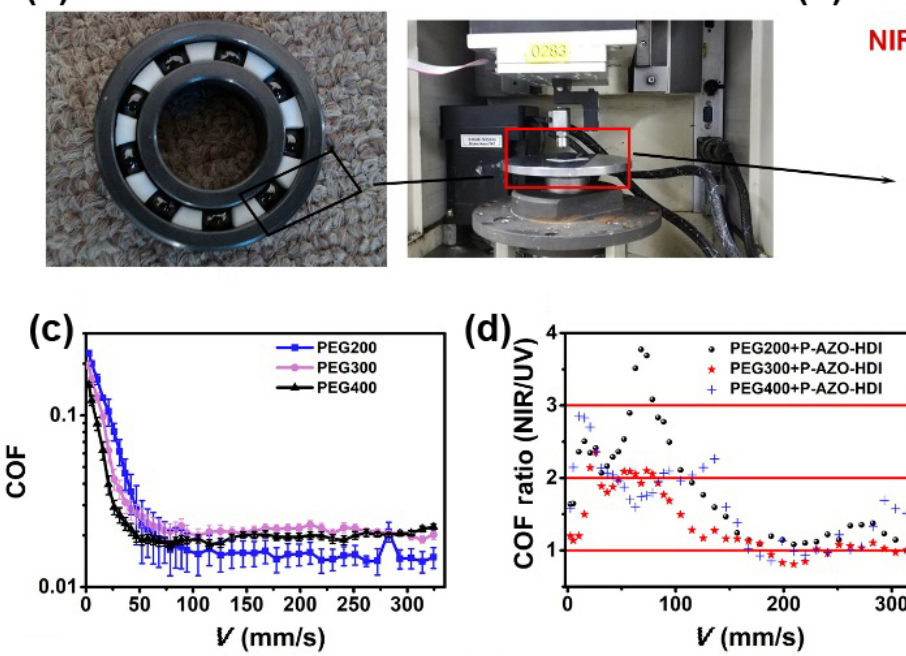

(f)

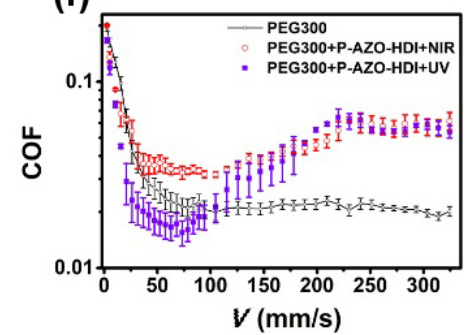

(d)

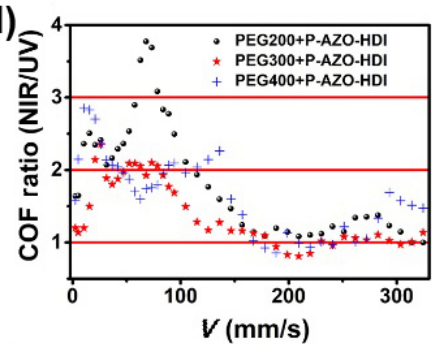

(g)

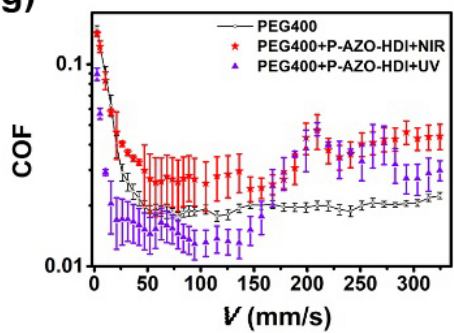

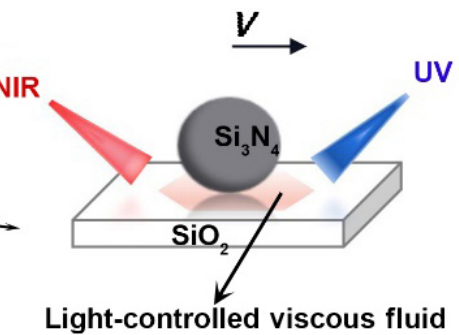

(e)

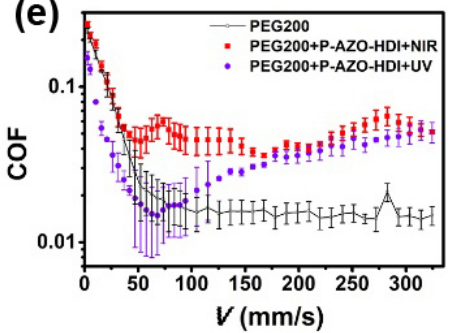

(h)

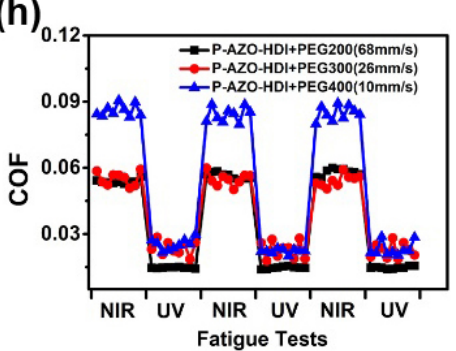

Fig. 5 Regulation of the COF for ceramic bearings by photosensitive rheological fluids. (a) Pictures of the ceramic sliding bearings and friction tests on UMT tribometers; (b) diagram of friction measurements regulated by UV/NIR light; (c) COF of pure PEG 200/300/400 solutions for varying test speeds; (d) COF ratio was defined by the COF under NIR light divided by that under UV light (The largest regulated ratios of the photosensitive rheological fluids were 3.77 in PEG200, 2.35 in PEG300, and 2.85 in PEG400); (e) COF of 2 wt\% P-AZO-HDI and PEG200 solutions for test speeds from 0 to $330 \mathrm{~mm} \cdot \mathrm{s}^{-1}$; (f) COF of $2 \mathrm{wt} \% \mathrm{P}-\mathrm{AZO}-\mathrm{HDI}$ and PEG300 solution for varying test speeds; (g) COF of $2 \mathrm{wt} \%$ P-AZO-HDI and PEG400 solution for varying test speeds; and (h) reversible friction regulation achieved by P-AZO-HDI and polyethylene glycol solutions (The COF tended to alternately change between $0.056 \pm 0.0098$ (NIR) and $0.015 \pm 0.0066$ (UV) at the speed of $68 \mathrm{~mm} \cdot \mathrm{s}^{-1}$ in PEG200 and $2 \mathrm{wt} \% \mathrm{P}-\mathrm{AZO}-\mathrm{HDI}$ solution, between $0.055 \pm 0.0076$ (NIR) and $0.023 \pm 0.0055$ (UV) at the speed of $26 \mathrm{~mm} \cdot \mathrm{s}^{-1}$ in PEG300 and $2 \mathrm{wt} \% \mathrm{P}-\mathrm{AZO}-\mathrm{HDI}$, and between $0.083 \pm 0.0084$ (NIR) and $0.029 \pm 0.0010$ (UV) at the speed of $10 \mathrm{~mm} \cdot \mathrm{s}^{-1}$ in PEG400 and $\left.2 \mathrm{wt} \% \mathrm{P}-\mathrm{AZO}-\mathrm{HDI}\right)$. 
and $2 \mathrm{wt} \%$ P-AZO-HDI changed from $0.055 \pm 0.0076$ irradiated by NIR light to $0.023 \pm 0.0055$ when exposed to UV light at the speed of $26 \mathrm{~mm} \cdot \mathrm{s}^{-1}$. The COF of PEG400 and $2 \mathrm{wt} \%$ P-AZO-HDI varied from $0.083 \pm 0.0084$ (NIR) to $0.029 \pm 0.0010$ (UV) at a speed of $10 \mathrm{~mm} \cdot \mathrm{s}^{-1}$. The results indicated that the photosensitive rheological fluids effectively regulated the friction of ceramic sliding bearings.

\subsection{Regulating mechanisms of light-controlled viscosity and friction}

According to the results of the simulations and experiments, the molecular structure differences before and after UV irradiation played a decisive role in the light-controlled viscosity. The trans isomer molecules under NIR light were long linear-chain structures, whereas the cis isomer molecules under UV light had serrated-chain morphologies, as shown in Fig. S3(f) in the ESM. The molecular length of the trans isomer was up to $33 \%$ longer than that of the cis isomer. The cone-plate viscosity measurement system was simplified as shown in Fig. 6(a). The higher viscosity was due to the greater possibility of twining on the vertical shear plane. Because the cis isomer of P-AZO-HDI had a serrated-chain morphology, a higher shear force is needed to overcome the resistance of the molecular tangle. In contrast, the trans isomer of P-AZO-HDI had a linear-chain structure and shear occurred easily due to almost no entanglement between these straight chains. This meant that the viscosity under NIR light was much smaller than that under UV light.

There were two critical factors, the photosensitivity of the rheological fluids and the mechanical properties
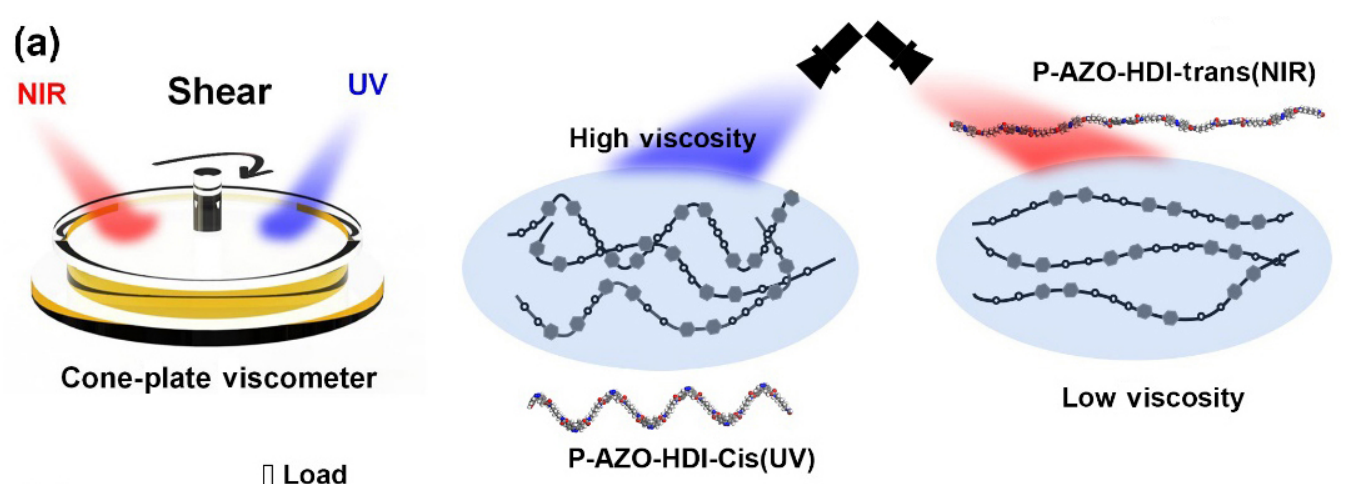

(b)
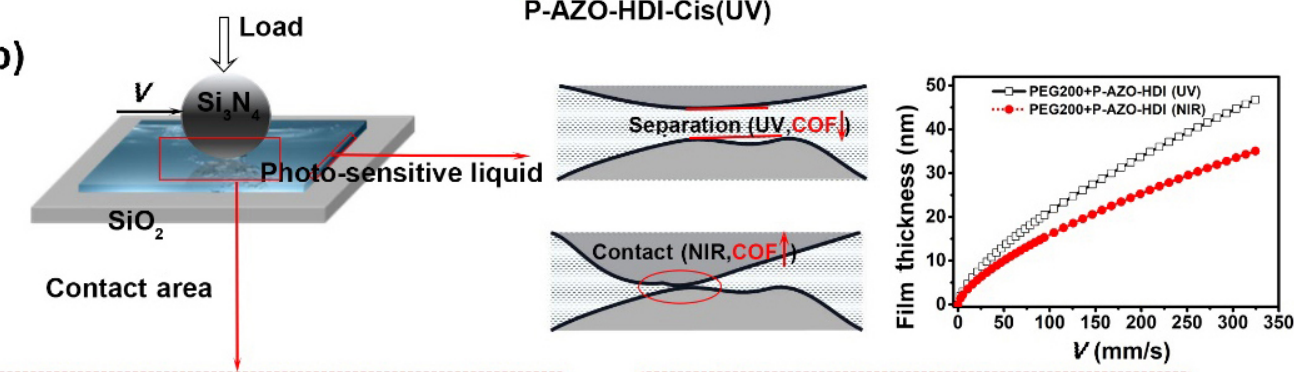

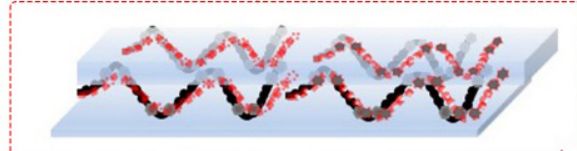

UV, bedding shear, easy to deform, COF $\downarrow$

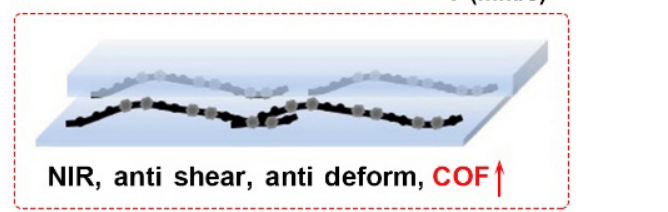

Fig. 6 Mechanisms of light-controlled viscosity and friction. (a) Light-controlled viscosity was ascribed to the structural differences between the trans and cis isomers of P-AZO-HDI (The P-AZO-HDI cis isomer was a serrated-chain structure, which meant that it was more likely to twine during shearing. The lower viscosity under NIR light was due to the linear-chain structure of the trans isomer) and (b) two factors (photosensitive fluids and mechanical properties of the P-AZO-HDI layer in contact areas) accounted for light-controlled friction (The higher viscosities (UV) meant thicker lubricated film. Therefore, frictional pairs were more likely to separate under UV light, which led to a lower COF. In addition, the P-AZO-HDI layer easily sheared and deformed, leading to a lower COF under UV light due to the smaller shear modulus and Young's modulus. In contrast, the higher COF under NIR light was due to the anti-shear and anti-deformation properties of the P-AZO-HDI layer with a larger shear modulus and Young's modulus. The photosensitive fluids and mechanical properties of the P-AZO-HDI layer worked together to regulate friction). 
of the P-AZO-HDI layer, which accounted for the friction regulation of ceramic sliding bearings. The mechanism of friction regulation (Fig. 6(b)) was divided into two parts. The first factor was the lubricated film thickness difference induced by the photosensitive fluid. In the boundary lubrication and the mixed lubrication (low speeds), the thickness of lubricated film has a significant influence on friction coefficient. According to Eq. (S6) in the ESM, the film thickness of $2 \mathrm{wt} \%$ P-AZO-HDI and PEG200 (Fig. 6(b)) under UV light was up to $30.2 \%$ thicker than that under NIR light. The lubrication film thickness ranges (Figs. S4(a) and S4(b) in the ESM) were up to $29.3 \%$ greater in the $2 \mathrm{wt} \%$ P-AZO-HDI and PEG300 solutions and up to $11.0 \%$ greater in the $2 \mathrm{wt} \%$ P-AZO-HDI and PEG400 solutions. The results indicated that the frictional pairs were more likely to be separated by a thicker film layer, leading to a smaller COF under UV light. The two surfaces were more likely to come into contact under NIR light, contributing to higher friction. When the speeds were higher than $180 \mathrm{~mm} \cdot \mathrm{s}^{-1}$, the lubricated state was elastohydrodynamic lubrication. The friction pair was separated by elastohydrodynamic fluid film, which led to the close friction coefficients under UV and NIR light.

The second factor was the mechanical property difference of the P-AZO-HDI layer on the contact areas. Here, the mechanical properties included the shear moduli and Young's moduli of the trans and cis isomers. As the results of the mechanical properties of P-AZO-HDI showed, the Young's modulus under NIR light was 2.62 times that of the modulus under UV light, as measured by AFM. The regulated range of the Young's modulus was 3.44 times according to the simulation of the mechanical properties. In addition, the simulated shear modulus under NIR light was 1.58 times that of the modulus under UV light. It was speculated that the P-AZO-HDI layer was hard to shear and deform with shearing direction due to the larger Young's modulus and shear modulus under NIR light. Therefore, the anti-shear and anti-deformation properties led to a higher COF under NIR light. When exposed to UV light, the P-AZO-HDI layer easily sheared and deformed with the shearing direction owing to the smaller Young's modulus and shear modulus. The bedding-shear and easy-to-deform
P-AZO-HDI layers resulted in smaller COFs under UV light. In conclusion, the photosensitive rheological fluids and mechanical properties of the P-AZO-HDI layer worked together to achieve light-controlled friction of ceramic sliding bearings.

\section{Conclusions}

In summary, we designed a group of photosensitive rheological fluids composed of $2 \mathrm{wt} \%$ poly $(4,4$ '-bishydroxyazobenzene hexamethylene diisocyanate) (P-AZO-HDI) and polyethylene glycol. These fluids can achieve reversible viscosity regulation via the obvious differences in the structural and mechanical properties under UV compared to NIR light. The P-AZO-HDI polymer with azobenzene units as the backbone was designed as a photoresponsive functional material. The molecular length under NIR light was $33 \%$ longer than that under UV light. The mechanical properties, such as the shear modulus and Young's modulus, were also significantly different under UV/NIR light. The regulated ranges of light-controlled viscosity were increased by $45.1 \%$ in PEG200, 43.8\% in PEG300, and $16.4 \%$ in PEG400. Photosensitive rheological fluids were used to achieve reversible friction regulation of ceramic sliding bearings. The maximum COF regulated ranges were 3.77 times greater in PEG200, 2.35 times greater in PEG300, and 2.85 times greater in PEG400. The mechanism of the light-controlled viscosity was ascribed to the structural differences between the trans and cis isomers of P-AZO-HDI. The friction regulation of ceramic bearings was due to the photosensitive fluids and mechanical properties of the P-AZO-HDI layer. The light-controlled viscosity, light-controlled shear modulus, and light-controlled Young's modulus worked together to regulate friction. This work not only provides a strategy to design photoresponsive polymers with quite different structures under UV/NIR irradiation but also develops new intelligent rheological fluids based on light stimuli. These results are significant for understanding the mechanism of viscosity and friction regulation from scientific and technological views. Photosensitive fluids have wide applications in smart rheological fluids, liquid transport, intelligent lubrication, and photoforce sensors. 


\section{Acknowledgements}

This work was financially supported by the National Natural Science Foundation of China (Nos. 51922058 and 51527901). We thank professor Shayu Li for the generous help on azobenzene polymer synthesis.

Electronic Supplementary Material: Supplementary Material is available in the online version of this article at https://doi.org/10.1007/s40544-021-0529-x.

Open Access This article is licensed under a Creative Commons Attribution 4.0 International License, which permits use, sharing, adaptation, distribution and reproduction in any medium or format, as long as you give appropriate credit to the original author(s) and the source, provide a link to the Creative Commons licence, and indicate if changes were made.

The images or other third party material in this article are included in the article's Creative Commons licence, unless indicated otherwise in a credit line to the material. If material is not included in the article's Creative Commons licence and your intended use is not permitted by statutory regulation or exceeds the permitted use, you will need to obtain permission directly from the copyright holder.

To view a copy of this licence, visit http://creativecommons.org/licenses/by/4.0/.

\section{References}

[1] Liu Y, Tan L. Method of C groove on vortex suppression and energy performance improvement for a NACA0009 hydrofoil with tip clearance in tidal energy. Energy 155: 448-461 (2018)

[2] Cheng H Y, Bai X R, Long X P, Ji B, Peng X X, Farhat M. Large eddy simulation of the tip-leakage cavitating flow with an insight on how cavitation influences vorticity and turbulence. Appl Math Model 77: 788-809 (2020)

[3] Lv J A, Liu Y Y, Wei J, Chen E Q, Qin L, Yu Y L. Photocontrol of fluid slugs in liquid crystal polymer microactuators. Nature 537(7619): 179 (2016)

[4] Ma L, Gaisinskaya-Kipnis A, Kampf N, Klein J. Origins of hydration lubrication. Nat Commun 6(1): 6060 (2015)

[5] Tang S, Xue D, Guo J, Ma L, Tian Y, Luo J. Macroscale Light-Controlled Lubrication Enabled by Introducing Diarylethene Molecules in a Nanoconfinement. Langmuir 36(21): 5820-5828 (2020)
[6] Martín-Alfonso J E, Martín-Alfonso M J, Valencia C, Cuberes M T. Rheological and tribological approaches as a tool for the development of sustainable lubricating greases based on nano-montmorillonite and castor oil. Friction 9(2): 415-428 (2021)

[7] Gao M, Li H, Ma L, Gao Y, Ma L, Luo J. Molecular behaviors in thin film lubrication-Part two: Direct observation of the molecular orientation near the solid surface. Friction 7(5): 479-488 (2019)

[8] Ma L, Luo J. Thin film lubrication in the past 20 years. Friction 4(4): 280-302 (2016)

[9] Jin Y, Chen F, Xu J, Yuan X. Nonlinear dynamic analysis of low viscosity fluid-lubricated tilting-pad journal bearing for different design parameters. Friction 8(5): 930-944 (2020)

[10] Jin Y, Shi Z, Zhang X, Yuan X. Rapid solution for analysis of nonlinear fluid film force and dynamic behavior of a tilting-pad journal bearing-rotor system with turbulent and thermal effects. Friction 8(2): 343-359 (2020)

[11] Wu Z. Multi-scale simulation of three-dimensional thin-film lubrication. Friction 9(3): 471-487 (2021)

[12] Liu J, Zang C, Tian S, Liu J, Yang H, Jia S, You L, Liu B, Zhang M. Water conservancy projects in China: Achievements, challenges and way forward. Glob Environ Change 23(3): 633-643 (2013)

[13] Li X, Dao M, Lykotrafitis G, Karniadakis G E. Biomechanics and biorheology of red blood cells in sickle cell anemia. J Biomech 50: 34-41 (2017)

[14] Ji X, Zhang W, Jia W, Wang X, Tian Y, Deng L, Liu J. Cactus-like double-shell structured $\mathrm{SiO}_{2} @ \mathrm{TiO}_{2}$ microspheres: Fabrication, electrorheological performances and microwave absorption. J Ind Eng Chem 56: 203-211 (2017)

[15] Liu C, Meng Y, Jia W, Zhang W, Zhao Q, Tian Y. Peanut shaped titanium oxide micro-particles achieved by cathode plasma electrolysis and their electrorheological characteristics. Smart Mater Struct 27(11): 115017 (2018)

[16] Yun G, Tang S-Y, Sun S, Yuan D, Zhao Q, Deng L, Yan S, Du H, Dickey M D, Li W. Liquid metal-filled magnetorheological elastomer with positive piezoconductivity. Nat Commun 10(1): 1300 (2019)

[17] Chen K, Shan L, Zhang X, Meng Y. Squeeze behaviors of magnetic powders between two parallel plates. Smart Mater Struct 23: 117004 (2014)

[18] Paul P S, Iasanth J A, Vasanth X A, Varadarajan A S. Effect of nanoparticles on the performance of magnetorheological fluid damper during hard turning process. Friction 3(4): 333-343 (2015)

[19] Li R, Li X, Li Y, Yang P-A, Liu J. Experimental and numerical study on surface roughness of magnetorheological elastomer for controllable friction. Friction 8(5): 917-929 (2020) 
[20] Lian C, Lee K-H, An J-W, Lee C-H. Effect of stick-slip on magneto-rheological elastomer with a magnetic field. Friction 5(4): 383-391 (2017)

[21] Chu Z L, Feng Y J. pH-switchable wormlike micelles. Chem Commun 46(47): 9028-9030 (2010)

[22] Lee H-Y, Diehn K K, Sun K, Chen T, Raghavan S R. Reversible photorheological fluids based on spiropyrandoped reverse micelles. J Am Chem Soc 133(22): 8461-8463 (2011)

[23] Yang D, Zhao J. A light-responsive organofluid based on reverse worm-like micelles formed from an equi-charged, mixed, anionic gemini surfactant with an azobenzene spacer and a cationic conventional surfactant. Soft Matter 12(17): 4044-4051 (2016)

[24] Chu Z, Dreiss C A, Feng Y. Smart wormlike micelles. Chem Soc Rev 42(17): 7174 (2013)

[25] Sparrow E M, Abraham J P, Gorman J M. Advances in Heat Transfer. San Diego(USA): Elsevier, 2018.

[26] Zhang X-D, Liu J. Perspective on liquid metal enabled space science and technology. Sci China Technol Sc 63(7): 1127-1140 (2020)

[27] Chen C, Liao W-H. A self-sensing magnetorheological damper with power generation. Smart Mater Struct 21(2): 025014 (2012)

[28] Aguirre N, Ikhouane F, Rodellar J, Christenson R. Parametric identification of the Dahl model for large scale MR dampers. Struct Control Health Monit 19(3): 332-347 (2012)

[29] Abramov V O, Abramova A V, Bayazitov V M, Mullakaev M S, Marnosov A V, Ildiyakov A V. Acoustic and sonochemical methods for altering the viscosity of oil during recovery and pipeline transportation. Ultrason Sonochem 35: 389-396 (2017)

[30] Abbadie A, Crescente F, SéMéRia M N. Advanced wet cleanings post-CMP. J Electrochem Soc 151(1): G57 (2004)

[31] Akagami Y, Asari K, Jeyadevan B, Fujita T. Characterization of particle motion for polishing and texturing under $\mathrm{AC}$ field by using Pprticle dispersion type ER fluid. J Intel Mat Syst Str 9(8): 672-675 (1998)

[32] Liu C, Meng Y, Tian Y. Potential-controlled boundary lubrication using $\mathrm{MoS}_{2}$ additives in diethyl succinate. Tribol Lett 68(3): 72 (2020)

[33] Amann T, Gatti F, Oberle N, Kailer A, Rühe J. Galvanically induced potentials to enable minimal tribochemical wear of stainless steel lubricated with sodium chloride and ionic liquid aqueous solution. Friction 6(2): 230-242 (2018)

[34] Zhang S, Qiao Y, Liu Y, Ma L, Luo J. Molecular behaviors in thin film lubrication - Part one: Film formation for different polarities of molecules. Friction 7(4): 372-387 (2019)

[35] Meng Y, Xu J, Jin Z, Prakash B, Hu Y. A review of recent advances in tribology. Friction 8(2): 221-300 (2020)
[36] Su J, Teng J, Xu Z, Li Y. Corrosion-wear behavior of a biocompatible magnesium matrix composite in simulated body fluid. Friction, 10(1): 31-43 (2022)

[37] Sun X, Guo R, Yuan B, Chen S, Wang H, Dou M, Liu J, He Z-Z. Low-temperature triggered shape transformation of liquid metal microdroplets. ACS Appl Mater Interfaces 12(34): 38386-38396 (2020)

[38] Groten J, Bunte C, Ruhe J. Light-induced switching of surfaces at wetting transitions through photoisomerization of polymer monolayers. Langmuir 28(42): 15038-15046 (2012)

[39] Han B, Zhang Y-L, Chen Q-D, Sun H-B. Carbon-based photothermal actuators. Adv Funct Mater 28(40): 1802235 (2018)

[40] Ichimura K, Oh S K, Nakagawa M. Light-driven motion of liquids on a photoresponsive surface. Science 288(5471): 1624-1626 (2000)

[41] Uyama A, Yamazoe S, Shigematsu S, Morimoto M, Yokojima S, Mayama H, Kojima Y, Nakamura S, Uchida K. Reversible photocontrol of surface wettability between hydrophilic and superhydrophobic surfaces on an asymmetric diarylethene solid surface. Langmuir 27(10): 6395-6400 (2011)

[42] Charati M B, Lee I, Hribar K C, Burdick J A. Lightsensitive polypeptide hydrogel and nanorod composites. Small 6(15): 1608-1611 (2010)

[43] Wani O M, Zeng H, Priimagi A. A light-driven artificial flytrap. Nat Commun 8:15546 (2017)

[44] Deng J, Li J, Chen P, Fang X, Sun X, Jiang Y, Weng W, Wang B, Peng H. Tunable photothermal actuators based on a pre-programmed aligned nanostructure. $\mathrm{J} \mathrm{Am} \mathrm{Chem} \mathrm{Soc}$ 138(1): 225-230 (2016)

[45] Pardo R, Zayat M, Levy D. Photochromic organic-inorganic hybrid materials. Chem Soc Rev 40(2): 672 (2011)

[46] Andréasson J, Pischel U. Molecules with a sense of logic: A progress report. Chem Soc Rev 44(5): 1053-1069 (2015)

[47] Li H, Wang J. Ultrafast yet controllable dual-responsive all-carbon actuators for implementing unusual mechanical movements. ACS Appl Mater Interfaces 11(10): 10218-10225 (2019)

[48] Wang S, Gao Y, Wei A, Xiao P, Liang Y, Lu W, Chen C, Zhang C, Yang G, Yao H, Chen T. Asymmetric elastoplasticity of stacked graphene assembly actualizes programmable untethered soft robotics. Nat Commun 11(1): 4359 (2020)

[49] Lee C K, Beiermann B A, Silberstein M N, Wang J, Moore J S, Sottos N R, Braun P V. Exploiting force sensitive spiropyrans as molecular level probes. Macromolecules 46(10): 3746-3752 (2013)

[50] Gao J, He Y, Xu H, Song B, Zhang X, Wang Z, Wang X. Azobenzene-containing supramolecular polymer films for laser-induced surface relief gratings. Chem Mater 19(1): 14-17 (2007) 
[51] Kumar G S, Neckers D C. Photochemistry of azobenzenecontaining polymers. Chem Rev 89(8): 1915-1925 (1989)

[52] Renth F, Siewertsen R, Temps F. Enhanced photoswitching and ultrafast dynamics in structurally modified photochromic fulgides. Int Rev Phys Chem 32(1): 1-38 (2013)

[53] Beharry A A, Woolley G A. Azobenzene photoswitches for biomolecules. Chem Soc Rev 40(8): 4422-4437 (2011)

[54] Ihalainen J A, Bredenbeck J, Pfister R, Helbing J, Chi L, van Stokkum I H M, Woolley G A, Hamm P. Folding and

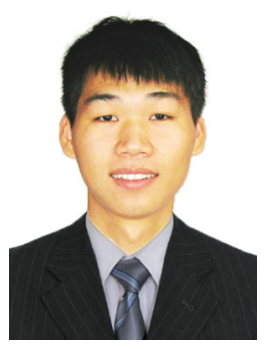

Shuangxi TANG. He obtained his bachelor degree in 2017 from University of Science and Technology Beijing. Then he was a Ph.D. student

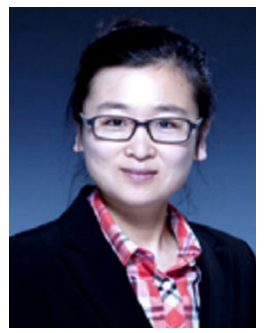

Liran MA. She received her bachelor degree from Tsinghua University in 2005, and received her Ph.D. degree from Tsinghua University in 2010. Following a postdoctoral period at the Weizmann Institute of Science in Israel, she is now working as an associate professor in State Key Laboratory of Tribology, Tsinghua University. Her interests in tribology have

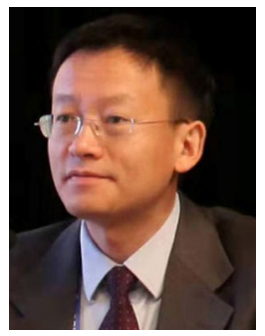

Yu TIAN. He is professor and director of the State Key Laboratory of Tribology at Tsinghua University of China. He gained his bachelor and Ph.D. degrees in mechanical engineering at Tsinghua University in 1998 and 2002, respectively. Subsequently he joined the State Key Laboratory of Tribology. He was a postdoc at the University of California, Santa Barbara with Professor Jacob Israelachvili from 2005 to 2007 . His research interest is the science and technology at the interface of physics, materials, engineering, and biology to understand the physical laws of adhesion, friction, and rheology to im- unfolding of a photoswitchable peptide from picoseconds to microseconds. P Natl Acad Sci USA 104(13): 5383 (2007)

[55] Nanni G, Ceseracciu L, Oropesa-Nunez R, Canale C, Salvatore P, Fragouli D, Athanassiou A. Tunable friction behavior of photochromic fibrillar surfaces. Langmuir 31(22): 6072-6077 (2015)

[56] Liu D, Broer D J. Light controlled friction at a liquid crystal polymer coating with switchable patterning. Soft Matter 10(40): 7952-7958 (2014)

at State Key Laboratory of Tribology of Tsinghua University. His research interests include lubrication mechanism and photorheological fluid.

ranged from aqueous lubrication and hydration lubrication to the liquid/solid interface properties. She has published over 50 papers. Her work has been cited some 300 times. Her honors include the Hinwin Doctoral Dissertation Award (2011), the Maple Leaf Award for Outstanding Young Iridologists (2015), and Chang Jiang Scholars Program-Young Professor Award (2015).

plement technological inventions to benefit the society. He has published over 150 peer-reviewed journal papers. He has received the Ten Thousand People Leading Plan Innovation Leading Talents of China (2019), the Youth Science and Technology Award of China (2016), the Yangtze River Scholars Distinguished Professor (2015-2019), the National Natural Science Foundation for Distinguished Young Scientists of China (2014), the Wen Shizhu-Maple Award-Young Scholar Award (2012), the Young Scholar Achievement Award of the Society of Mechanical Engineering of China (2011), Outstanding Young Scholar Award of the Chinese Tribology Institute (2009), and the National Excellent Doctoral Dissertation of China (2004). 